\title{
Scanning Microbeam X-Ray Scattering of Fibers Analyzed by One-Dimensional Tomography
}

\author{
Norbert Stribeck,* Ulrich Nöchel, Armando Almendárez Camarillo
}

The investigation of structure gradients in polymer fibers or pipes by the X-ray microbeam scanning technique is put on its theoretical fundament. The inverse Abel transform desmears measured data in X-ray scattering fiber computer-tomography (XSF-CT). Fast, low noise algorithms from one-dimensional tomography are available. They are applicable to scan data in which the X-ray absorption, the small-angle X-ray scattering (SAXS), or the wide-angle X-ray diffraction (WAXD) is measured. The method is demonstrated by application to SAXS scan data from a polymer fiber. The resulting sequence of image-space SAXS patterns is reflecting the nanostructure variation along the fiber radius.

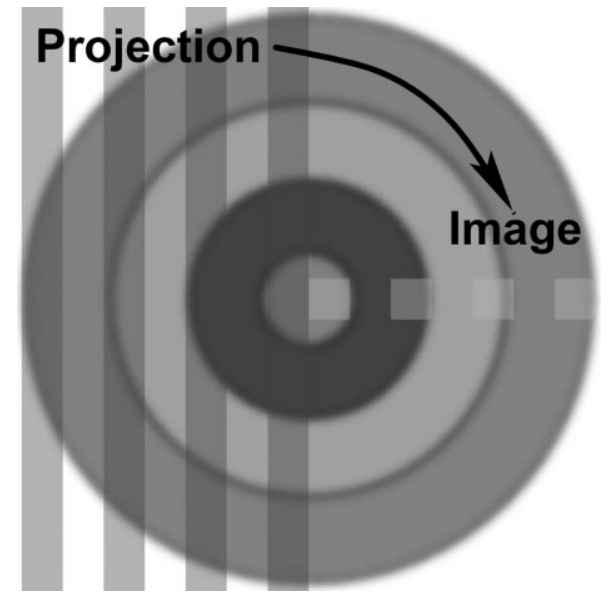

\section{Introduction}

Products with cylindrical symmetry are frequently investigated in applied polymer science. An important example are fibers. Their structure is studied by X-ray scattering since this method has been devised. ${ }^{[1-4]}$ Continuous scientific interest is reflected in the fact that nowadays fibers are frequently studied by the application of an X-ray microbeam scanning technique. ${ }^{[5-19]}$ In this process, the fiber is irradiated in the direction perpendicular to the fiber axis by a fine X-ray beam that is stepwise translated across the fiber. The result is a series of absorption values or of projected scattering patterns, which vary as a function of

N. Stribeck, U. Nöchel

Department of Chemistry, Institute of Technical and

Macromolecular Chemistry, University of Hamburg,

Bundesstr. 45, 20146 Hamburg, Germany

Fax: (+49) 4042838 6008; E-mail: norbert.stribeck@desy.de

A. A. Camarillo

Instituto Tecnológico de Celaya, Av. Tecnológico y A. García

Cubas, 38010 Celaya, Gto., Mexico the scan position $x$ on the fiber diameter. The variation is interpreted and qualitatively related to structure variation along the fiber radius.

In a general SAXS tomography study, ${ }^{[20]}$ we have pointed out the shortcoming of such interpretation, which has clearly been stated in a paper of Paris et al. ${ }^{[21] ~ " t h e ~}$ long-term goal is to proceed from microbeam scanning experiments to a real imaging technique". In the general case, experiment and image reconstruction are still time consuming. Routine studies of structure gradients in polymer materials are not yet possible. The situation is much more favorable, if fibers or pipes are studied. A complete set of low-noise scattering patterns can be collected in less than $30 \mathrm{~min}$. Thus, an application-oriented $\mathrm{X}$-ray analytics with spatial resolution for fibers is already available. Nevertheless, for routine application the computational effort for image reconstruction must be decreased considerably. In fact, image reconstruction, as well, should be much easier than in the case of general tomography. In many of the microbeam scanning studies published during the last decade, a complete set of projection data for a tomographic experiment has been 
collected, but the image reconstruction has been omitted. The considerable potential of information increase after tomographic imaging is well known. Sporadic efforts to solve the image reconstruction problem in X-ray scattering stay rudimentary. This is astonishing, because the mathematically isomorphous problem of slit smearing and its solution is exemplified in many textbooks of X-ray scattering. ${ }^{[22-28]}$ Nevertheless, it appears inappropriate to re-activate the classical tools of slit-desmearing, because they are noisy and slow. An illumination considering the progress made in other fields of science with the numerical solution of the same problem is more adequate. This study is dedicated to the introduction of a suitable reconstruction method for materials with fiber symmetry in the field of polymer analytics; namely the X-ray scattering fiber computer-tomography (XSF-CT).

Compared to common tomographic imaging, the XSFCT does not arbitrarily choose an imaging function which must be matched for the purpose of optimum contrast visualization of certain structural features. Because no imaging function is chosen, there is no need to validate the reconstruction itself. Imaging is pure mathematics. In fact, this mathematics is equivalent to the well-known slit-desmearing which has extensively been applied in scattering decades ago, when slit cameras (Kratky camera, Rigaku-Denki camera) were used to compensate the low intensity of former X-ray sources. Moreover, here each voxel in the fiber is not simply represented by a gray value (zero-dimensional scalar number), but by a complete scattering pattern (three-dimensional function). On the one hand, this means that the reconstruction cannot be presented in a gray-scale picture without arbitrarily choosing an imaging function. On the other hand, the scattering pattern contains the complete information on the nanostructure from inside its voxel - provided the scattering pattern itself exhibits (local) fiber symmetry.

\section{Theoretical Review}

In a general tomographic X-ray experiment, ${ }^{[29]}$ a voluminous sample is scanned by a thin X-ray beam. As a function both of the position $x$ of the scanning beam on the sample, and of the sample rotation angle $\phi$, projections (notation: \{\}$)$ of the absorption $\{A\}(\chi, \phi)$ or even of complete scattering patterns $\{I\}(\mathbf{s}, \chi, \phi)$ are measured, in order to analyze the structure variation in the plane of the sample that is scanned by the X-ray beam. ${ }^{[20,30,31]}$ Here, $\mathbf{s}$ is the scattering vector with $|\mathbf{s}|=s=(2 / \lambda) \sin \theta$, the X-ray wavelength $\lambda$, and the scattering angle $2 \theta$. In the examples mentioned, a tomographic image reconstruction ${ }^{[29,32,33]}$ returns either the spatial variation of the absorption in the plane, or of the scattering emanating from small volume elements (voxels) in the plane. The smearing caused from projection is eliminated by application of the Fourier transform theory, and a clear image of the inner structure is obtained.

If the studied material shows cylindrical symmetry, the results of the measurement are no function of $\phi$ any more, and the complete image information is in a single microbeam scan. The fundamental geometry is sketched in Figure 1 . The information in the measured signal $\{A\}(x)$ or $\{I\}(\mathbf{s}, x)$, respectively does not represent the sought information $\{A\}(x)$ or $\{I\}(\mathbf{s}, x)$ originating from the small square (voxel) around the position $x$. Instead, to a first approximation it is represented by the projection integral

$$
\begin{aligned}
\{I\}(\mathbf{s}, x) & =2 \int_{x}^{\infty} I\left(\mathbf{s}, \sqrt{\chi^{2}+y^{2}}\right) d y \\
& =2 \int_{x}^{\infty} \frac{I\left(\mathbf{s}, \rho_{f}\right) \rho_{f} d \rho_{f}}{\sqrt{\rho_{f}^{2}-\chi^{2}}}
\end{aligned}
$$

In the equation only the accumulated attenuation of the primary beam by X-ray absorption is not accounted for. The sought information in image space $\left(I\left(\rho_{f}\right)\right)$ along the radius $\rho_{f}$ of the fiber has to be reconstructed from the information in projection space $(\{I\}(\chi))$.

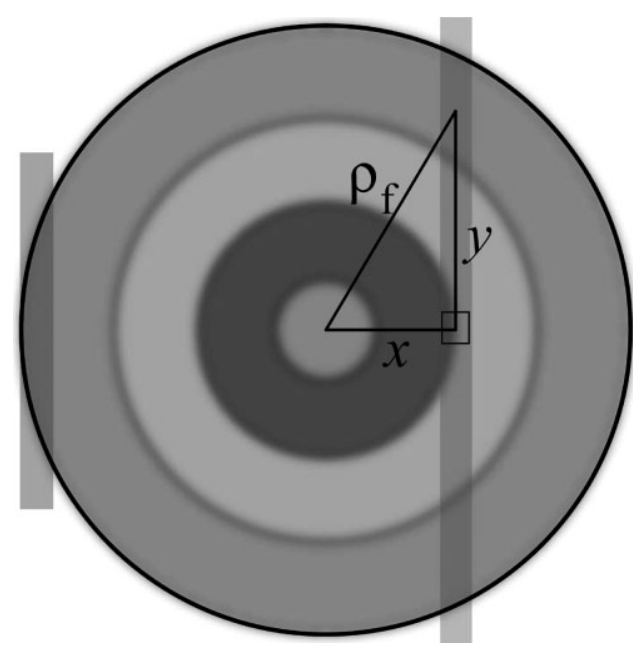

Figure 1. A fiber is irradiated by an X-ray microbeam (long vertical bar) at an offset $x$ from its center. The structure $\rho\left(\rho_{f}\right)$ inside the fiber shows fiber symmetry. The $X$-ray beam is probing a superposition (integration, projection) of all the structures that are interacting with the beam. $y$ is the variable of the integration. Close to the fiber surface (short vertical bar) the recorded data are not subjected to superposition. 
Equation (1) is the definition of the Abel transform. ${ }^{[33]}$ In X-ray scattering Equation (1) is established textbook knowledge. ${ }^{[22-28]}$ There it describes the slit smearing. Even the inverse Abel transform

$$
\begin{aligned}
I(\mathbf{s}, x) & =-\frac{1}{\pi} \int_{0}^{\infty} \frac{d\{I\}\left(\mathbf{s}, \rho_{f}\right)}{d \rho_{f}} \frac{d y}{\rho_{f}} \\
& =-\frac{1}{\pi} \int_{x}^{\infty} \frac{d\{I\}\left(\mathbf{s}, \rho_{f}\right)}{d \rho_{f}} \frac{d \rho_{f}}{\sqrt{\rho_{f}^{2}-\chi^{2}}},
\end{aligned}
$$

which first has been derived by Niels Abel ${ }^{[34]}$ is found in scattering textbooks since Guinier ${ }^{[35]}$ and DuMond. ${ }^{[36]}$ Thus, fiber symmetry simplifies tomography considerably. Instead of a reconstruction by filtered backprojection, ${ }^{[32,33]}$ the inverse Abel transform according to Equation (3) or Equation (4) suffices. It must be mentioned that, here, we implicitly assume that the scattering from every irradiated voxel in the fiber shows fiber symmetry itself (local fiber symmetry). Otherwise reconstruction errors ${ }^{[20]}$ are expected.

Scattering curves from the Kratky camera, ${ }^{[37]}$ have frequently been desmeared by application of Equation (3). For that purpose, the measured data must be smoothed and derived in the direction of $\rho_{f .}{ }^{[38]}$ In order to avoid derivation, loss of a background, and generation of excess noise, several iterative numerical algorithms have been devised. ${ }^{[39-45]}$ They are fast enough for the desmearing of a few scattering curves. On the other hand, several direct methods have been proposed, ${ }^{[46,47]}$ which interpret the smearing integral of Equation (1) by a system of linear equations which are solved by matrix inversion using Gauss-Jordan elimination. The main problem with this approach is the control of noise in the result. ${ }^{[4]}$

In other fields of science, different and more effective solutions have been developed in the last decades. A frequent pedestrian method for the inversion of Equation (1) starts from the discussion of the case, in which the probing beam is just touching the fiber (in Figure 1 demonstrated on the left side of the fiber plane). In this case, the recorded signal contains only the information on the outmost shell that cannot be resolved any further. Thus, this shell can be peeled off. According to Equation (2) the contribution of an infinitesimal thin layer

$$
I(x)=\delta\left(x-\rho_{f}\right)
$$

at the position $\rho_{f}$ to the projection is (cf. also Bracewell ${ }^{[33]}$ p. 354)

$$
\{I\}(x)=\frac{2 \rho_{f}}{\sqrt{\rho_{f}^{2}-\chi^{2}}} Y_{\rho_{f}}(x),
$$

with $Y_{r}(x)=1$ for $|x|<r$ and $Y_{r}(x)=0$ elsewhere being the symmetric shape function of width $2 r$. After respective subtraction, the shell beneath has been excavated and is ready for peeling. This method is called onion peeling technique or topological thinning and is used in several fields (cf. citations in Bitter et al. ${ }^{[48]}$ ).

More powerful and accurate algorithms for the computation of the inverse Abel transform are continuously enhanced in the one-dimensional tomography, i.e., the tomography of objects with cylindrical symmetry. ${ }^{[49,50]}$ In principle, these algorithms first compute a general reconstruction matrix, $\mathbf{R}$, which does not need to be inverted any more. Thereafter, the reconstruction of the projection-space signal $\{I\}$ to the image space is simply accomplished by

$$
I=\mathbf{R}\{I\}
$$

multiplication of the projection-space vector with the reconstruction matrix. Because of this general property there are no principal differences of numerical effort, if different algorithms are compared. The art of matrix setup is in the consideration of the derivative from Equation (4). Dasch $^{[49]}$ is approximating the derivative in a two-point Abel inversion by linear differences. The three-point Abel inversion is based on polynomial approximation. In the laboratory of Reisler, ${ }^{[50]}$ the BASEX method has been developed. In this method, every voxel in image space is represented by a Gaussian basic function with the width of a voxel. The representation of each Gaussian in projection space is analytical [Reisler ${ }^{[50]}$, Equation (15)], based on Equation (5). Thus, the projection space data are expanded in terms of these basis functions without the need to perform approximated derivatives during the computation of $\mathbf{R}$. The result is a detailed and low-noise reconstruction.

It is recommended to scan the fiber with constant step size and to carry out the reconstruction in relative units of this step size. The index 0 is associated to the image obtained when irradiating the center of the fiber. Under these premises the reconstruction matrices $\mathbf{R}$ can be used universally. Once a matrix has been computed and stored for a hypothetical scan with a narrow step size, it can be reused over and over again. If a coarse-grid experiment has been carried out, the reconstruction matrix is simply cropped to fit the length of the voxel-vector along the fiber radius.

For example, if a fiber scan comprises 11 scattering patterns, the radius contains six elements labeled by the indices $0-5$. Then, a precomputed reconstruction matrix $\mathrm{rmp}$ yields the required matrix $\mathrm{rm}$ by cropping $r m=r m p(0: 5,0: 5)$. Then, for the BASEX algorithm the elements of $\mathrm{rm}$ are: 


\begin{tabular}{cccccc}
0.753745 & -0.203849 & 0.0182095 & -0.00168307 & $1.52606 \mathrm{e}-4$ & $-1.34885 \mathrm{e}-05$ \\
-0.693183 & 0.572202 & -0.0661508 & 0.00625179 & $-5.67699 \mathrm{e}-4$ & $5.01819 \mathrm{e}-05$ \\
0.0694336 & -0.363726 & 0.336305 & -0.0355081 & 0.00324849 & $-2.87272 \mathrm{e}-4$ \\
-0.0711409 & 0.0772521 & -0.272953 & 0.271834 & -0.0266249 & 0.00236337 \\
$8.02250 \mathrm{e}-4$ & -0.0501747 & 0.0617679 & -0.228969 & 0.235275 & -0.0220235 \\
-0.0161177 & 0.00644846 & -0.0428576 & 0.0563194 & -0.201629 & 0.210617 \\
\hline
\end{tabular}

Although it might not be obvious at the first glance, the resulting sequence of scattering patterns forms, in fact, a tomographic image of the fiber. Rotation about the central point generates the two-dimensional image of the crosssectional plane (annular rings), and extrusion of this plane in the direction of the fiber axis yields the threedimensional image of the nanostructure variation inside the fiber.

\section{Experimental Part}

\section{Material}

The studied polymer material is a microfibrillar reinforced composite $^{[51]}$ (MFC) containing poly(ethylene terephthalate) (PET) microfibrils embedded in a poly(ether)-block-amide (PEBAX) matrix (composition: PEBAX/PET 70:30 wt.-\%). After co-extrusion the material has been cold-drawn to a draw ratio $\lambda_{d} \approx 3$.

\section{Setup}

Scanning microbeam SAXS experiments are carried out at HASYLAB, Hamburg, beamline BW4. The incident primary beam (wavelength $\lambda=0.13 \mathrm{~nm}$ ) is focused by means of a stack of Belenses yielding a beam cross-section at the sample of $40 \mu \mathrm{m}$ integral width and $39 \mu \mathrm{m}$ height as measured by a knife edge. The demonstration sample is a cylindrical strand of $1.95 \mathrm{~mm}$ diameter. The material is linearly scanned through the beam with a step size of $50 \mu \mathrm{m}$. The distance between sample and detector is $1910 \mathrm{~mm}$. Each scattering pattern is exposed for $40 \mathrm{~s}$ using a 2-D marCCD 165 detector (mar research, Norderstedt, Germany). A low-noise machine background pattern is exposed for $3 \mathrm{~min}$. The absorption of the primary beam is measured by monitoring the beam intensity before and after the sample.

A simple check for fiber symmetry has been carried out by performing a second scan after rotating the fiber by $90^{\circ}$ about its axis and comparing the results. The scans are identical.

Due to the long exposure the signal on the detector is high above the noise level. The typical intensity of the equatorial streak is 20000 counts above a background level of 50. A ring-shaped reflection observed in all patterns shows a maximum of 2000 counts even in the center of the fiber where it is the weakest.

\section{Pre-Evaluation}

The measured machine background pattern is subtracted from each scan pattern after weighting by the absorption factor. No normalization to constant sample thickness is performed. Further, pre-evaluation steps follow the standard method. ${ }^{[28]}$ The images are centered and aligned, some blind spots can be filled from symmetry consideration. The remnant hole in the center is filled by extrapolation. A border area is cut off, in order to obtain quadratic scattering patterns of valid pixels. The pre-evaluated scattering patterns hold data in the interval $-0.2 \mathrm{~nm}^{-1}<s_{12}$, $s_{3}<0.2 \mathrm{~nm}^{-1}$. Here, $s=\sqrt{s_{12}^{2}+s_{3}^{2}}$ is the modulus of the scattering vector $\mathbf{s}$ with $s=(2 / \lambda) \sin \theta .2 \theta$ is the scattering angle. $s_{12}$ and $s_{3}$ are the equatorial and the meridional (fiber axis) components, respectively. The generation of a valid-pixel mask and the determination of the penumbra region around the blind spots takes $2 \mathrm{~h}$. The pre-evaluation of each image is manually controlled and the processing of a series of 39 patterns takes $10 \mathrm{~min}$.

\section{Preparation of Image Reconstruction}

For image reconstruction, the center of the fiber has to be determined. The first image of the input data series has to be an image from the exact center of the fiber. For this purpose, interpolation between the recorded images is carried out. By finetuning of the center, the series from the left side of the fiber can be made identical to the series from the right side.

Because of the size of each scattering pattern $(666 \times 666$ pixels), neither the whole pattern stack measured in projection space, nor the whole image stack of reconstructed images can be kept in the computer memory. Thus, we keep input and output tile stacks in memory with each tile comprising only $32 \times 32$ pixels. Most of the time of the reconstruction ( $6 \mathrm{~min}$ ) is spent by the tile management (moving tile contents between memory and disk).

Then, the reconstruction work according to Equation (6) is accomplished in one line of source code:

$$
\operatorname{tsr}(x i, y i, *)=\operatorname{rm} \# \operatorname{REFORM}(\operatorname{tsi}(x i, y i, *), n)
$$

tsi(xi, yi, $\left.{ }^{*}\right)$ is piercing $\left(^{*}\right)$ through the input-tile stack tsi at the position xi, yi. The input tile stack is $n$ patterns high. $\operatorname{REFORM}(\ldots, n)$ makes the collected pixel data a vector, which is matrix-multiplied (\#) to the $(n \times n)$ reconstruction matrix $\mathrm{rm}$ to yield the reconstructed pixels in the tile stack tsr. Reconstruction algorithms are only differing by the elements of the reconstruction matrix.

\section{Reconstruction Matrices}

Following the paper of Dasch, ${ }^{[49]}$ we tried to compute the reconstruction matrices related to the two-point and the threepoint Abel inversion method. The published equations of the 
three-point inversion contain several errors (indices pointing outside the matrix, square roots of negative numbers). The reported two-point Abel inversion method contains only one typo which could be corrected. In Dasch's Equation (9) the denominator in the bottom line must be changed to

$$
\left(j^{2}-i^{2}\right)^{1 / 2}+j .
$$

After the correction, the matrix setup algorithm is producing the elements of the reconstruction matrix reported by Dasch. Moreover, we have implemented the BASEX ${ }^{[50]}$ method.

\section{Results}

Both the BASEX algorithm and the two-point Abel inversion return essentially the same results. Only in the center of the fiber differences are found, which can even be seen in a logarithmic scaling of the scattering intensities (Figure 2).

Compared to a typical direct matrix inversion method developed in X-ray scattering, ${ }^{[46]}$ already the two-point Abel inversion is a considerable improvement, because the returned noise is sufficiently low. Nevertheless, the BASEX method is even much less noisy (cf. Figure 3 ) if it is applied to the low-noise scattering data of the test sample. Thus, both methods appear suitable to reconstruct SAXS data. Moreover, by means of the BASEX algorithm it should even be possible to reconstruct WAXD data with narrow peaks.

Figure 4 shows the result of the demonstration sample in three bands of double-rows. In the representation, each pattern is individually scaled to its maximum intensity. The upper row of each band displays the

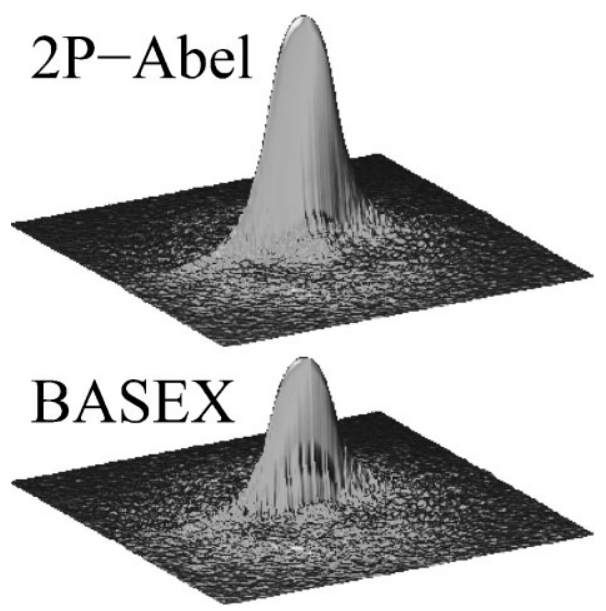

Figure 2. Results of one-dimensional tomography. Comparison of reconstruction in the fiber center. The two-point Abel inversion (top) predicts more remnant scattering than the BASEX method (bottom). The scattering patterns display the range $-0.1 \mathrm{~nm}^{-1}<s_{12}, s_{3}<0.1 \mathrm{~nm}^{-1}$. Logarithmic intensity scaling.

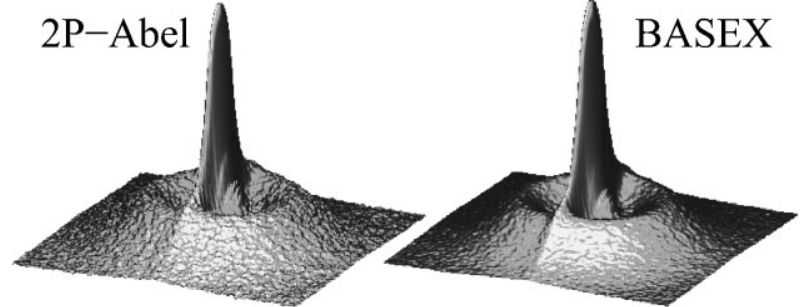

Figure 3. Results of one-dimensional tomography. Comparison of reconstruction algorithms. The two-point Abel inversion (left) is more noisy than the BASEX method (right). SAXS scattering patterns from a volume element taken at half the fiber radius. The scattering patterns display the range $-0.1 \mathrm{~nm}^{-1}<s_{12}$, $s_{3}<0.1 \mathrm{~nm}^{-1}$.

projections, $\{I\}\left(s_{12}, s_{3}, \rho_{f}\right)$, measured when looking through the fiber at the position $\rho_{f}$. The lower row of each band reports the corresponding reconstructed scattering patterns, $I\left(s_{12}, s_{3}, \rho_{f}\right)$, in image space. Every pattern describes the scattering emanating from a small voxel at the
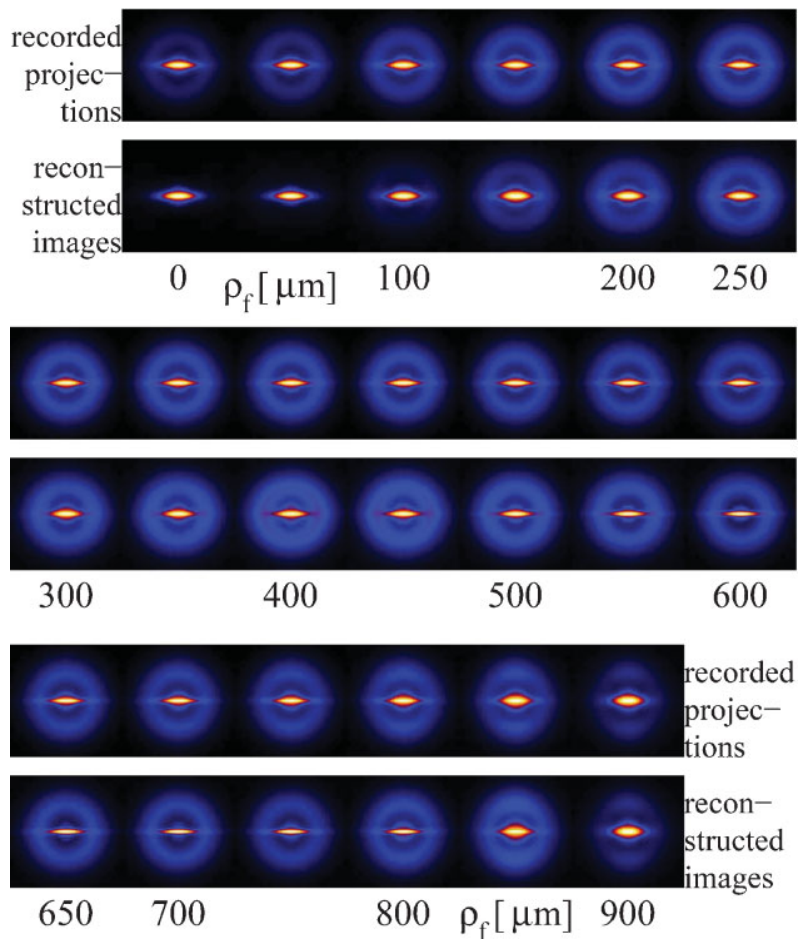

Figure 4. One-dimensional tomography in practice. The upper rows show the measured scattering patterns, $\{l\}\left(s_{12}, s_{3}, \rho_{f}\right)$, from a scanning microbeam experiment as a function of the scan position $\rho_{f}$ with respect to the fiber center (logarithmic intensity scale). The lower rows display the reconstructed scattering patterns, I $\left(s_{12}, s_{3}, \rho_{f}\right)$ as a function of a position $\rho_{f}$ on a fiber radius. The plots display only the central part of the patterns in the range $-0.1 \mathrm{~nm}^{-1}<s_{12}, s_{3}<0.1 \mathrm{~nm}^{-1}$. The fiber axis, $s_{3}$, is vertical. 
position $\rho_{f}$ on the fiber radius. The perturbing information from volume elements before and behind the central voxels has been eliminated by tomographic reconstruction. Consequently, the reconstructed images contain clear quantitative information on the variation of the nanostructure along the fiber radius.

The recorded scattering patterns of the material show two peculiar features. In the center of each pattern there is an equatorial streak extending in horizontal direction. It can be related to the PET microfibrils embedded in the material. The second feature is a diffuse ring reflection related to a long period in the PEBAX block copolymer matrix. In the projection-space patterns the two features can hardly be separated with respect to their position inside the fiber. Only the reconstructed images clearly show that the ring-shaped feature is completely missing in the center of the fiber. In image space, the indication of a long period is first visible at an offset $\rho_{f}$ of $100 \mu \mathrm{m}$ from the fiber axis. With increase in offset a widening of the ring and a broadening of the reflection indicates a decrease in the average long period and a widening of the long period distribution. At the fiber surface the diffuse reflection shows maxima in the (vertical) fiber direction. In the measured projections this orientation is observed from $\rho_{f}=800 \mu \mathrm{m}$ outward. Nevertheless, the reconstructed patterns reveal that, actually, the hard domain orientation is limited to a thin zone on the fiber surface around $\rho_{f}=900 \mu \mathrm{m}$. As the fiber surface is approached from the center, the projection space information shows a continuous widening of the equatorial streak. Inspection of the image-space patterns reveals that, in fact, the streak is suddenly widening at about $\rho_{f}=850 \mu \mathrm{m}$.

\section{Conclusion}

The method of one-dimensional tomography that is applied in X-ray scattering for the first time, is implemented with low experimental and numerical effort. Whereas, the experimental microbeam technique has been considerably advanced in recent decades, ${ }^{[7]}$ the methodical aspect has so far been insufficiently considered. This is demonstrated by many studies in which microbeam scans of fibers are interpreted without resorting to desmearing. The undoubted academic value of such studies can be turned into quantitative and practical value, if analytical methods are developed which are established in the theory. As has been shown by our study, a fundamental solution of the analytical problem is obvious. By application of the adapted method clear, significant, and quantitative data concerning the structure gradient in a textile fiber can be obtained. However, for this purpose the use of a synchrotron beamline which is dedicated for microbeam applications is recommended. For the pre- sented material reconstruction is perfect. This is due to the fact that the scattering from every single voxel shows fiber symmetry. If this premise is not fulfilled, reconstruction aberrations will show up. How such aberrations can be utilized to gain deeper understanding of the nanostructure will be reported in a follow-up study.

Comparing the power of the available direct reconstruction algorithms to the early desmearing algorithms from $\mathrm{X}$-ray scattering, reassessment of the potential of the direct desmearing methods is indicated. As demonstrated in tomography, the direct algorithms are not necessarily returning noisy results.

Acknowledgements: We wish to thank A. Timmann (HASYLAB), M. Kuhlmann (HASYLAB), C. Schroer (University of Dresden, Germany), and J. Feldkamp (University of Dresden, Germany) for their support with the measurement. In particular, we thank H. Reisler and B. Karpichev (University Los Angeles, USA) for their support with the implementation of the BASEX method and S. Fakirov (University of Auckland, New Zealand) for the material. We acknowledge HASYLAB, Hamburg, for provision of the synchrotron radiation facilities at beamline BW4 in the frame of project II-04-039. Financial support by the Deutsche Forschungsgemeinschaft (DFG STR501/4-2) is gratefully acknowledged.

Received: May 8, 2008; Revised: July 8, 2008; Accepted: July 9 , 2008; DOI: $10.1002 /$ macp.200800242

Keywords: fibers, microstructure, microtomography (micro-CT), scanning microbeam, small-angle X-ray scattering (SAXS)

[1] O. Kratky, Kolloid Z. 1933, 64, 213.

[2] O. Kratky, Kolloid Z. 1933, 68, 347.

[3] O. Kratky, Naturwiss. 1938, 26, 94.

[4] P. H. Hermans, P. Platzek, Kolloid-Z. 1939, 88, 68.

[5] Y. Kajiura, S. Watanabe, T. Itou, A. Iida, Y. Shinohara, Y. Amemiya, J. Appl. Cryst. 2005, 38, 420.

[6] C. Riekel, P. Engstrom, C. Martin, J. Macromol. Sci. Phys. 1998, B37, 587.

[7] C. Riekel, Rep. Prog. Phys. 2000, 63, 233.

[8] C. Riekel, M. C. García Gutiérrez, A. Gourrier, S. V. Roth, Anal. Bioanal. Chem 2003, 376, 594.

[9] Y. Nozue, Y. Shinohara, Y. Amemiya, Polym. J. 2007, 39, 1221.

[10] M. Müller, C. Czihak, G. Vogl, P. Fratzl, H. Schober, C. Riekel, Macromolecules 1998, 31, 3953.

[11] Y. D. Wang, M. Cakmak, Polymer 2001, 42, 4233.

[12] O. Paris, D. Loidl, M. Müller, H. Lichtenegger, H. Peterlik, J. Appl. Cryst. 2001, 34, 473.

[13] D. Loidl, H. Peterlik, O. Paris, M. Müller, M. Burghammer, C. Riekel, J. Synchrotron. Radiat. 2005, 12, 758.

[14] O. Paris, D. Loidl, H. Peterlik, M. Müller, H. Lichtenegger, P. Fratzl, J. Appl. Cryst. 2000, 33, 695.

[15] O. Paris, D. Loidl, H. Peterlik, Carbon 2002, 40, 551.

[16] M. Müller, C. Czihak, M. Burghammer, C. Riekel, J. Appl. Cryst. 2000, 33, 817. 
[17] M. Müller, C. Riekel, R. Vuong, H. Chanzy, Polymer 2000, 41, 2627.

[18] D. Lozano-Castelló, E. Raymundo-Piñero, D. Cazorla-Amorós, A. Linares-Solano, M. Müller, C. Riekel, Carbon 2002, 40, 2727.

[19] A. Flores, A. Poeppel, C. Riekel, K. Schulte, J. Macromol. Sci. Phys. 2001, 40, 749.

[20] N. Stribeck, A. Almendarez Camarillo, U. Nöchel, C. Schroer, M. Kuhlmann, S. V. Roth, R. Gehrke, R. K. Bayer, Macromol. Chem. Phys. 2006, 207, 1239.

[21] O. Paris, C. Li, S. Siegel, G. Weseloh, F. Emmerling, H. Riesemeier, A. Erko, P. Fratzl, J. Appl. Cryst. 2007, 40, s466.

[22] A. Guinier, G. Fournet, Small-Angle Scattering of X-Rays, Chapman and Hall, London 1955.

[23] R. Hosemann, S. N. Bagchi, Direct Analysis of Diffraction by Matter, North-Holland, Amsterdam 1962.

[24] L. E. Alexander, X-Ray Diffraction Methods in Polymer Science, Wiley, New York 1979.

[25] O. Glatter, O. Kratky, Eds., Small Angle X-ray Scattering, Academic Press, London 1982.

[26] L. A. Feigin, D. I. Svergun, Structure Analysis by Small-Angle $X$-Ray and Neutron Scattering, Plenum Press, New York 1987.

[27] F. J. Baltá Calleja, C. G. Vonk, X-Ray Scattering of Synthetic Polymers, Elsevier, Amsterdam 1989.

[28] N. Stribeck, X-Ray Scattering of Soft Matter, Springer, Heidelberg, New York 2007.

[29] U. Bonse, F. Busch, Prog. Biophys. Mol. Biol. 1996, 65, 133.

[30] C. G. Schroer, M. Kuhlmann, S. V. Roth, R. Gehrke, N. Stribeck, A. Almendarez Camarillo, B. Lengeler, Appl. Phys. Lett. 2006, 88, 164102.

[31] C. G. Schroer, M. Kuhlmann, T. F. Günzler, B. Benner, O. Kurapova, J. Patommel, B. Lengeler, S. V. Roth, R. Gehrke,
A. Snigirev, I. Snigireva, N. Stribeck, A. Almendárez Camarillo, F. Beckmann, Proc. Soc. Photo Opt. Instrum. Eng. 2006, 6318, 6318.

[32] A. C. Kak, M. Slaney, Principles of Computerized Tomographic Imaging, IEEE Press, New York 1999.

[33] R. Bracewell, The Fourier Transform and Its Applications, $3^{\text {rd }}$ edition, Mc Graw-Hill, New York 1999.

[34] N. H. Abel, J. Reine Angew. Math. 1826, 1, 153.

[35] A. Guinier, G. Fournet, Nature 1947, 160, 501.

[36] J. W. M. DuMond, Phys. Rev. 1947, 72, 83.

[37] O. Kratky, G. Porod, L. Kahovec, Z. Elektrochem. 1951, 55, 53.

[38] J. J. Müller, E. C. Müller, C. Gernat, R. Kröber, J. Appl. Cryst. 1995, 28, 38

[39] V. Synecek, Acta Cryst. 1960, 13, 378.

[40] G. R. Strobl, Acta Cryst. 1970, A26, 367.

[41] O. Glatter, J. Appl. Cryst. 1974, 7, 147.

[42] C. G. Vonk, J. Appl. Cryst. 1975, 8, 340.

[43] M. Deutsch, M. Luban, J. Appl. Cryst. 1978, 11, 87.

[44] M. Deutsch, M. Luban, J. Appl. Cryst. 1978, 11, 98.

[45] V. Synecek, Acta Cryst. 1962, 15, 642.

[46] C. G. Vonk, J. Appl. Cryst. 1971, 4, 340.

[47] M. H. Singh, S. S. Ghosh, R. F. Shannon, Jr., J. Appl. Cryst. 1993, 26, 787.

[48] I. Bitter, A. E. Kaufman, M. Sato, IEEE Trans. Vis. Comput. Graph. 2001, 7, 195.

[49] C. J. Dasch, Appl. Opt. 1992, 31, 1146.

[50] V. Dribinsi, A. Ossadtchi, V. A. Mandelshtam, H. Reisler, Rev. Sci. Instrum. 2002, 73, 2634.

[51] M. Evstatiev, S. Fakirov, B. Krasteva, K. Friedrich, J. A. Covas, A. M. Cunha, Polym. Eng. Sci. 2002, 42, 826. 